\title{
A ADAPTABILIDADE DE SOLUÇÕES MOM COMO FATOR ESTRATÉGICO*
}

\section{Resumo}

\author{
Alexandre Keunecke Hardt ${ }^{1}$ \\ Giuliano Felippe Soares ${ }^{1}$ \\ Marcelo Cardoso Martins Vieira ${ }^{2}$ \\ Roberto Resque de Freitas $^{2}$
}

A alta competitividade no setor siderúrgico aliada ao dinamismo crescente do mercado internacional tem forçado as empresas a estarem mais preparadas para eventuais necessidades de mudanças em seus processos e produtos. Em muitas situações, o tempo de resposta de uma empresa para adaptar-se a uma nova necessidade, estratégia ou tecnologia determina o sucesso ou fracasso da iniciativa. As soluções MOM, como responsáveis pelo gerenciamento da produção, possuem um papel fundamental no intuito de facilitar a adaptação da empresa a uma nova realidade. Nesse contexto, a adaptabilidade e a flexibilidade das ferramentas MOM tornam-se fatores extremamente relevantes no momento de definição de um produto ou concepção de uma solução. Este trabalho tem como objetivo refletir sobre o papel das soluções MOM como acelerador de mudanças dentro das indústrias. Serão apresentadas as características principais de um sistema MOM preparado para o cenário dinâmico atual, bem como alguns critérios para avaliação da adaptabilidade e flexibilidade de uma solução MOM.

Palavras-chave: MES; MOM; Adaptabilidade; Flexibilidade; Diferenciação Competitiva;

\section{THE ADAPTABILITY OF MOM SYSTEMS AS A STRATEGIC FACTOR Abstract}

The very competitive steel market along with the dynamic international markets have pushed the need for the companies to be more prepared for quick changes in their processes and products. In many situations, the response time for a company to adapt to a new requirement, strategy or technology will determine its success or failure in that initiative. The MOM systems, with their responsibility to manage manufacturing, play a fundamental role to support the company in adapting to new scenarios. In this context, the adaptability and flexibility of the MOM applications become really relevant when defining which product to use or in the conception of a solution. This paper has the objective to analyze the role of MOM systems as a change enablement accelerator in the companies. The main characteristics of a flexible solution will be presented along with some criteria to evaluate the adaptability and flexibility of the MOM systems.

Keywords: MES; MOM; Adaptability; Flexibility; Competitive differentiation.

1 Engenheiro de Controle e Automação, Consultor de sistemas MES da Accenture do Brasil LTDA, Belo Horizonte, MG, Brasil.

2 Engenheiro de Controle e Automação, Gerente de projetos MES da Accenture do Brasil LTDA, Belo Horizonte, MG, Brasil.

* Contribuição técnica ao $18^{\circ}$ Seminário de Automação e TI Industrial, 23 a 26 de setembro de 2014, São Paulo, SP, Brasil. 


\section{INTRODUÇÃO}

As indústrias siderúrgicas e de mineração no Brasil, assim como no resto do mundo, estão diante de uma nova e bastante desafiadora realidade. Se por um lado a globalização do mercado e a internacionalização das empresas criaram diversas novas oportunidades de crescimento, por outro lado acarretou em um mercado ainda mais competitivo e em nível mundial.

As grandes empresas estão buscando cada vez mais aperfeiçoarem os seus processos produtivos e de gestão operacional no intuito de conquistar um diferencial competitivo que represente uma redução de custos, melhoria da qualidade ou ainda redução no prazo de entrega dos seus produtos. Os clientes, sejam consumidores ou corporações, estão demandando cada vez mais de seus fornecedores e esse fenômeno ocorre em todas as partes do mundo. Suas demandas precisam ser atendidas no menor tempo possível, com a maior qualidade possível e, mais do que nunca, de forma personalizada [1]. Essa realidade força as empresas a terem respostas ainda mais rápidas às necessidades de mudanças em seus processos e produtos para atender a este cenário. Tais mudanças podem ser motivadas por diferentes iniciativas, tais como melhorias de processo, adequação a novas regulamentações, adaptações para atendimento de novos mercados, padronização de soluções corporativas, evolução de sistemas de automação, adaptações devido à volatilidade do mercado, entre outros inúmeros fatores. Em muitas situações inclusive, a capacidade da empresa de se adaptar rapidamente a uma nova demanda determina o seu sucesso em uma nova oportunidade, sobretudo no tocante a decisões estratégicas que envolvem pioneirismo em produtos e mercados ou necessidade de adaptação a uma nova realidade.

Nesse cenário, as soluções MOM [1] tem um papel fundamental nos processos de adaptação a novas situações, uma vez que tipicamente é a porção mais maleável ou flexível da pirâmide de informação industrial (Figura 1).

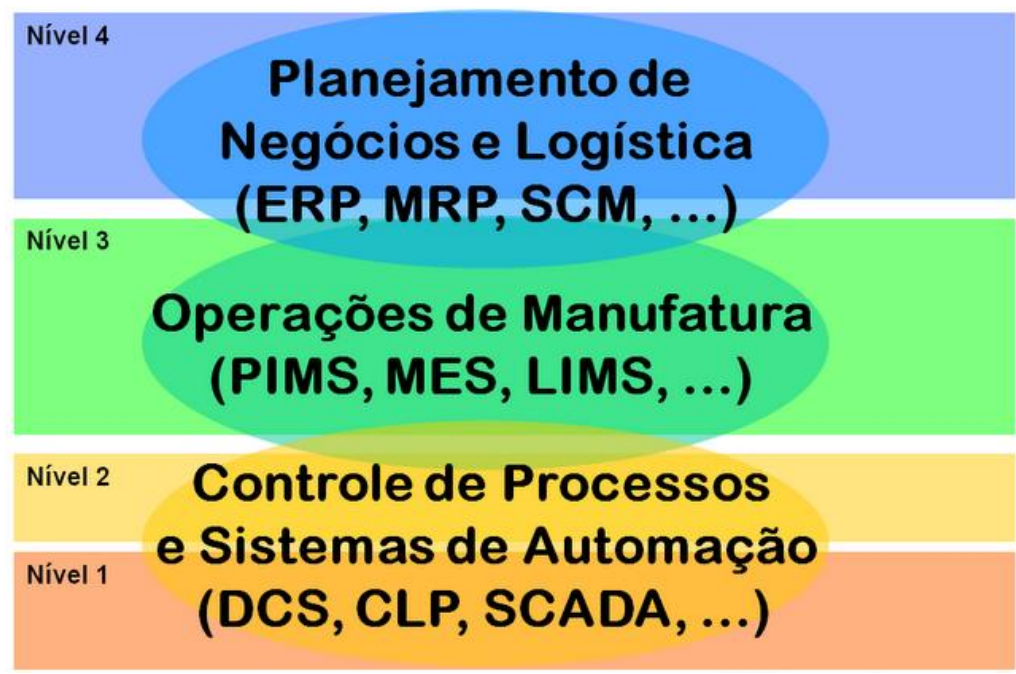

Figura 1. Níveis da Pirâmide de Informação [2-4].

O nível 4 da pirâmide (ERP) é muitas vezes uma solução global e padrão, que nem sempre está aberta a modificações mais profundas (por inúmeros motivos, entre eles o próprio caráter de padronização desses sistemas). Já os níveis 1 e 2 (PLC e Supervisórios) estão diretamente acoplados às características físicas do processo e muitas vezes estão fundamentados em tecnologias antigas e de difícil modificação. Os sistemas de nível 3 geralmente requerem a capacidade de serem flexíveis de

* Contribuição técnica ao $18^{\circ}$ Seminário de Automação e TI Industrial, 23 a 26 de setembro de 2014, São Paulo, SP, Brasil. 
forma a atender à padronização corporativa, mas ao mesmo tempo se conectarem aos sistemas industriais. Essa posição intermediária requer que os sistemas MOM tenham a capacidade de se adaptar ao processo, sendo geralmente o local apropriado para absorver o dinamismo de mudanças na produção.

Em outras palavras, é comum a ferramenta MOM ficar responsável por absorver algumas necessidades de mudanças na execução do processo produtivo e as mesmas devem responder de forma satisfatória às demandas com uma boa adaptabilidade/flexibilidade para tais mudanças.

O objetivo deste artigo é discutir a relevância da adaptabilidade e flexibilidade das soluções MOM no contexto atual das indústrias siderúrgicas e de mineração. Serão apresentados e detalhados alguns critérios relevantes para uma análise de flexibilidade e adaptabilidade.

\section{ABORDAGEM PARA AVALIAÇÃO DA ADAPTABILIDADE}

A adaptabilidade é uma característica qualitativa para soluções MOM, ou seja, não é possível avaliar quantitativamente a adaptabilidade de uma solução MOM, uma vez que tal avaliação considera alguns fatores complexos, difíceis de serem mensurados e que dependem da circunstância/aplicação da solução. No intuito de tentar uma melhor assertividade na avaliação da adaptabilidade, a análise pode ser realizada com base em alguns critérios bem definidos. Tal abordagem visa guiar o leitor em uma análise pautada em critérios objetivos, o que possibilita evitar uma analise tendenciosa de fornecedores de soluções MOM.

Em um grau mais ou menos elevado e com uma complexidade maior ou menor, grande parte das soluções de mercado e até mesmo sistemas custom/bespoke são classificados como satisfatoriamente ou altamente adaptáveis pelos seus fornecedores. Essa classificação é embasada algumas vezes por padrões consolidados de mercado, seja de alguma tecnologia ou órgão internacional. Um exemplo comum é o alinhamento do modelo de dados de algumas soluções com o padrão ANSI/ISA-95 [2-4]. Pode-se afirmar que o padrão ANSI/ISA-95 garante uma boa adaptabilidade a uma ferramenta MOM? O alinhamento à ISA-95 certamente é um passo importante nessa direção, mas ele sozinho não consegue garantir a flexibilidade requerida ao sistema. Apesar de o modelo de dados proposto pelo padrão ANSI/ISA-95 ser extremamente genérico e com capacidade para atender processos produtivos distintos de diversas indústrias dos mais variados segmentos, existem outros fatores relevantes a serem considerados em uma análise de adaptabilidade, tais como a tecnologia/plataforma utilizada pela ferramenta e a arquitetura da solução, por exemplo.

No exemplo em questão, uma solução MOM pode ter um modelo de dados/entidades muito aderente ao padrão ANSI/ISA-95. Porém, um requisito não funcional, tal como a falta de suporte ao controle de versão da solução, pode reduzir consideravelmente a adaptabilidade da ferramenta, sobretudo em um cenário cuja aplicação tenha âmbito nacional ou global. Isso porque uma vez implantada a solução em diversas plantas ao redor do mundo, uma modificação um pouco mais profunda para atender uma nova demanda pode afetar seriamente diversas unidades industriais. A falta de uma funcionalidade de controle de versão de alterações irá dificultar em muito o processo de alteração, teste e implantação dessas modificações.

O capítulo a seguir apresenta algumas características importantes a serem consideradas em uma análise da adaptabilidade de uma ferramenta MOM. Os

* Contribuição técnica ao $18^{\circ}$ Seminário de Automação e TI Industrial, 23 a 26 de setembro de 2014, São Paulo, SP, Brasil. 
fatores devem ser analisados individualmente. Contudo, o conjunto dos fatores é que confere um caráter mais ou menos adaptável a solução. É importante ressaltar que os critérios apresentados neste trabalho constituem-se uma referência para uma avaliação padrão de uma solução MOM. A iniciativa para a qual a solução está sendo concebida pode trazer algumas outras características relevantes a serem consideradas e, portanto, entender claramente o cenário é fundamental para a análise.

\section{CRITÉRIOS PARA AVALIAÇÃO DA ADAPTABILIDADE DE SOLUÇÕES MOM}

\subsection{Alinhamento com o Padrão ANSI/ISA-95}

O padrão internacional ANSI/ISA-95 divulgado pela ISA (Internacional Society of Automation) foi elaborado com o objetivo de ser aplicável a todas as indústrias nos seus diversos tipos de processos e de naturezas distintas: batelada, contínuo e discreto. O padrão define modelos, terminologias e funções padrão para sistemas de nível 3 da pirâmide industrial, além de interfaces com os níveis 2 e 4 . Por se tratar de um padrão internacional de grande aceitação no mercado e pela qualidade comprovada das definições apresentadas no padrão, sem dúvida alguma 0 alinhamento da ferramenta MOM com os termos e principalmente com o modelo de dados/entidades e atributos definidos pela norma é um fator que confere adaptabilidade à solução.

Para exemplificar a importância desse fator, podemos analisar uma situação real. A Figura 2 apresenta o modelo de entidades de materiais definido pelo padrão ANSI/ISA-95 - Parte 2. Como pode ser observado, o modelo é bastante completo e genérico. Sendo assim, o modelo de dados/entidades de uma ferramenta MOM que estiver alinhado com o padrão sugerido pela norma provavelmente atenderá a diversas necessidades de adaptações que porventura venham a surgir.

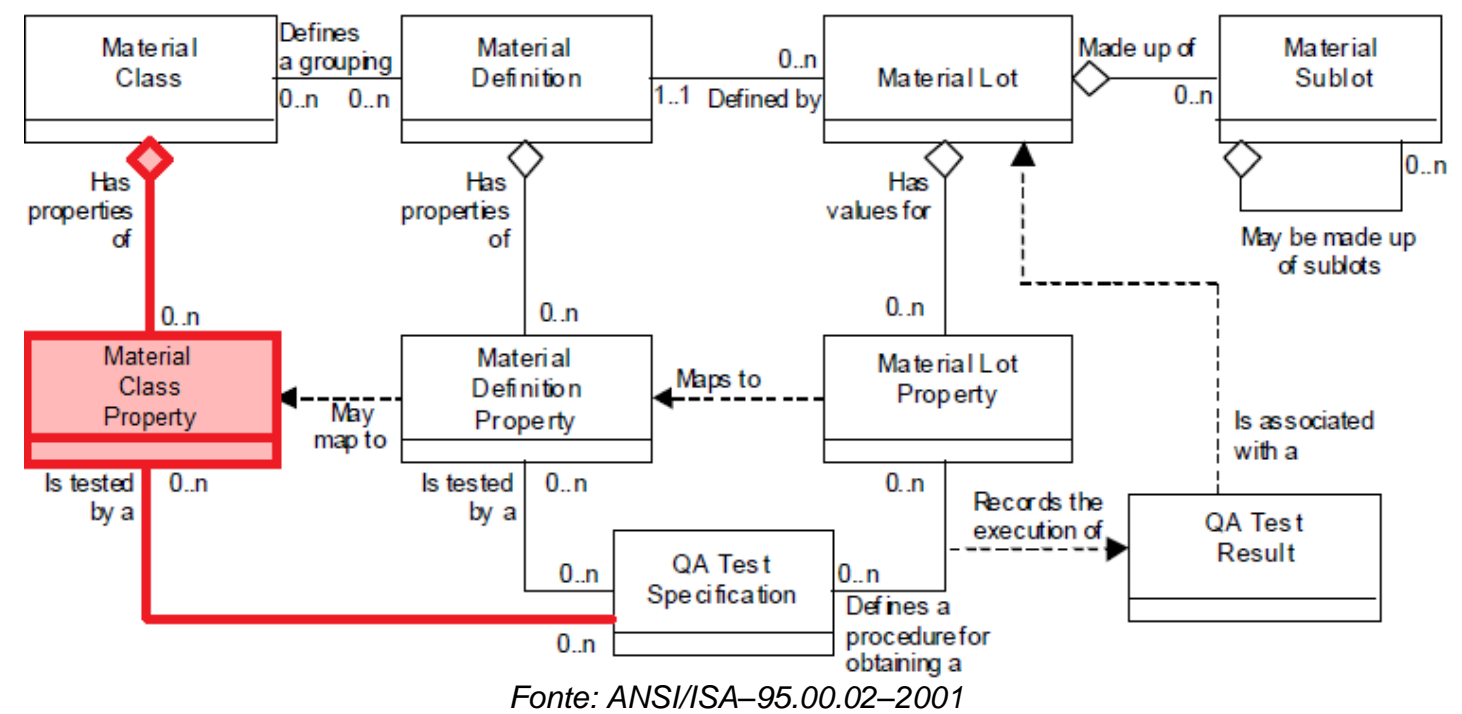

Figura 2. Modelo de materiais definido pela ANSI/ISA-95

Suponhamos que uma determinada solução/ferramenta não esteja completamente alinhada com o modelo de materiais do padrão ANSI/ISA-95 e não exista a possibilidade de associar propriedades a classes de materiais, conforme apresentado na Figura 2. Ou seja, durante a concepção da ferramenta não foi criada

* Contribuição técnica ao $18^{\circ}$ Seminário de Automação e TI Industrial, 23 a 26 de setembro de 2014, São Paulo, SP, Brasil. 
a entidade de propriedades de classes de materiais, pois o cenário até então era de que todas as propriedades estavam diretamente associadas ao material.

Em um momento futuro, após a implantação da solução em diversas plantas, é identificada a necessidade de adaptar a solução para padronizar globalmente os dados mestres de materiais, motivado, por exemplo, por um projeto de padronização global da camada corporativa. Nesse caso, a necessidade de associar propriedades a classes de materiais poderia simplificar bastante o processo de adaptação da ferramenta, reduzindo o custo, tempo e prazo da alteração no sistema, além de significar uma simplificação e eliminação de redundância nos dados.

Portanto, a aderência do modelo de entidades/dados de uma solução MOM com o padrão definido na ANSI/ISA-95 fomenta a adaptabilidade da ferramenta, por se tratar de um padrão internacional elaborado por representantes de diversas indústrias dos mais variados segmentos e que é de qualidade reconhecida no mercado.

\subsection{Tecnologia/Plataforma}

A tecnologia ou plataforma em que a ferramenta foi construída é também um fator muito relevante para ser levado em consideração ao avaliar a adaptabilidade de uma solução MOM.

Tecnologias de desenvolvimento de sistemas em geral, como Java e .NET, são naturalmente flexíveis, uma vez que não são específicas para sistemas de manufatura. Essas tecnologias são geralmente usadas no desenvolvimento de soluções MOM custom ou plataformas de suporte para tal desenvolvimento. Nesse caso, a adaptabilidade da solução final será diretamente dependente dos conceitos de desenvolvimento de software que forem utilizados durante a concepção do sistema.

Por outro lado, os produtos MOM disponíveis no mercado possuem diferentes formas de customização que devem ser analisadas para determinar se a solução será flexível ou não. Mesmo grande parte desses produtos sendo desenvolvido nas tecnologias citadas anteriormente, geralmente o código-fonte não é disponibilizado para modificações e/ou elas devem ser feitas em ambientes específicos de customização. Essa capacidade de customização tem uma grande variabilidade entre os diferentes produtos disponíveis no mercado e essa característica deve ser analisada para verificar se tal produto poderá ser facilmente modificado de forma a se adaptar às mudanças dos processos produtivos e corporativos ou não.

\subsection{Controle de Versão}

Os sistemas de controle de versão são extremamente úteis no processo de desenvolvimento de software, pois permitem o desenvolvimento controlado de diferentes versões de uma mesma aplicação. Com isso, é possível trabalhar simultaneamente no desenvolvimento de melhorias e em ajustes pontuais da solução, assim como facilitar o roll-out / upgrade da solução para outras plantas.

Nas áreas de ciência da computação e sistemas de informação, o suporte de tecnologias e ferramentas a sistemas de controle de versão é algo intrínseco em praticamente todos os casos: uma tecnologia já bem dominada e amplamente difundida. No entanto, no campo de ferramentas MOM essa ainda não é uma realidade. Alguns produtos de "prateleira" de grandes players do mercado ainda não

* Contribuição técnica ao $18^{\circ}$ Seminário de Automação e TI Industrial, 23 a 26 de setembro de 2014, São Paulo, SP, Brasil. 
possuem um suporte integrado a sistemas de controle de versão e dificilmente permitem o uso de um sistema externo para fazer este papel.

Ao pensar em adaptabilidade de soluções industriais, deve-se considerar que ao mesmo tempo em que a ferramenta está passando por um processo de mudança, ela estará sendo utilizada no ambiente produtivo das inúmeras plantas onde a solução já estiver implantada e com uma missão crítica para a produção. Por esse motivo, é fundamental que as modificações sejam controladas, possibilitando assim o desenvolvimento de novas funcionalidades em paralelo com a manutenção do sistema no ambiente produtivo sem expor a ferramenta a uma situação de risco para as unidades operacionais.

\subsection{Interoperabilidade}

Antes de abordar a interoperabilidade das ferramentas MOM como fator relevante na análise de adaptabilidade, é importante definir o que está sendo considerado como interoperabilidade neste trabalho. A interoperabilidade é a capacidade de um sistema de integrar-se facilmente com outros sistemas.

Como as soluções MOM ocupam a porção intermediária da pirâmide de informação industrial é inevitável que a aplicação tenha que trocar informações com outros sistemas, como, por exemplo, Supervisórios, PIMS, LIMS, ERP, entre outros inúmeros casos. Sendo assim, a facilidade para integrar a ferramenta com outros sistemas é um fator muito relevante na análise de adaptabilidade.

As principais características de uma solução MOM a ser avaliada em uma análise de interoperabilidade são:

- Arquitetura de comunicação: qual a arquitetura de comunicação da ferramenta (SOA, preferencialmente);

- Disponibilidade de conectores: quais os conectores padrões já disponibilizados pela ferramenta (web services, arquivo texto, conexões a banco de dados, entre outros);

- Monitoramento de interfaces: quais as ferramentas disponibilizadas pela aplicação para monitoramento de interfaces (logs, histórico de execuções, etc.). Portanto, a facilidade com que a ferramenta pode ser integrada ou conectada a um sistema externo é também um fator importante na análise de adaptabilidade da solução.

\subsection{Internacionalização}

Considerando a abrangência normalmente global de empresas de siderurgia e mineração, o critério de internacionalização da solução MOM é um fator muito relevante em termos de adaptabilidade. Algumas características importantes são descritas abaixo:

- Suporte multi-idiomas: a aplicação deve ser configurável de forma a permitir que o usuário escolha o idioma que será exibido. Além disso, deve ser possível traduzir facilmente a aplicação para novos idiomas;

- Formato de datas e números: considerando que usuários de países diferentes usarão o sistema, deve ser possível configurar o formato de data, como por exemplo, mês/dia/ano em vez de dia/mês/ano, assim como o formato de números, como os separadores de decimal e de milhar;

- Suporte a múltiplas unidades de medida: devido principalmente à utilização dos sistemas métricos e imperial em diferentes países, o suporte a diferentes

\footnotetext{
* Contribuição técnica ao $18^{\circ}$ Seminário de Automação e TI Industrial, 23 a 26 de setembro de 2014,
} São Paulo, SP, Brasil. 
unidades de medida e a conversão desses valores para exibição de acordo com a escolha do usuário é fundamental. Além disso, o uso de unidades de medidas diferentes está relacionado a praticas de determinados mercados ou mesmo requisitos normativos como emissão de certificados.

\subsection{Arquitetura em Camadas}

Uma solução MOM flexível deve ser formada por uma camada de apresentação (telas, interfaces com o usuário, coletores, tablets), uma camada de lógica (onde estão as regras de negócio) e uma camada de persistência (onde as informações são armazenadas), além da camada de integração citada na sessão 0.3 .4 Interoperabilidade.

Essas camadas devem ser desacopladas, ou seja, deve ser possível evoluir a tecnologia utilizada em uma camada sem afetar as demais. Por exemplo, uma aplicação MOM flexível deve permitir a consulta de informações pelo navegador de internet, assim como pelo celular. Da mesma forma, em determinada planta a solução pode utilizar um sistema de banco de dados e em outra planta utilizar outro sistema diferente.

\section{CONCLUSÃO}

Seja devido a uma nova estratégia de negócio, seja por uma demanda do mercado, ou até uma alteração no processo produtivo, as mudanças enfrentadas pelas indústrias de siderurgia e de mineração estão se tornando cada vez mais frequentes, fazendo com que a velocidade de adaptação a essas mudanças seja uma vantagem competitiva.

Como sistemas responsáveis pela gestão da produção, as soluções MOM dessas indústrias precisam se mostrar flexíveis e adaptáveis de forma a facilitar a execução da mudança. Porém, saber o nível de adaptabilidade de um sistema MOM não é uma questão apenas de identificar um valor numa escala de 0 a 10 . Existem fatores não quantificáveis que devem ser levados em consideração ao se avaliar a adaptabilidade e a flexibilidade.

Portanto, o artigo procurou expor os critérios mais relevantes, explicando a importância e o impacto de cada critério, inclusive com exemplos, para avaliar a adaptabilidade e a flexibilidade de uma solução MOM.

\section{REFERÊNCIAS}

1 Is Your MES Flexible Enough To Change As Requirements Evolve?. Critical Manufacturing. 2014 - [acesso em 06 mai. 2014]. Disponível em:

http://www.criticalmanufacturing.com/en/newsroom/blog/posts/blog/is-your-mesflexible-enough-to-change-as-requirements-evolve

2 ISA. (s.d.). Enterprise-Control System Integration: Part 1: Models and Terminology. ANSI/ISA-S95.00.01-2000. ISBN: 1-55617-727-5: 2000.

3 ISA. (s.d.). Enterprise-Control System Integration: Part 2: Object Model Attributes. ANSI/ISA-95.00.02-2001. ISBN: 1-55617-773-9: 2001.

4 ISA. (s.d.). Enterprise-Control System Integration: Part3: Activity Models of Manufacturing Operations Management. ANSI/ISA-95.00.03-2005. ISBN: 1-55617-9553: 2005.

* Contribuição técnica ao $18^{\circ}$ Seminário de Automação e TI Industrial, 23 a 26 de setembro de 2014, São Paulo, SP, Brasil. 